\title{
Many-body dynamics in long-range hopping models in the presence of correlated and uncorrelated disorder
}

\author{
Ranjan Modak ${ }^{1}$ and Tanay $\mathrm{Nag}^{2}$ \\ ${ }^{1}$ SISSA and INFN, via Bonomea 265, 34136 Trieste, Italy \\ ${ }^{2}$ SISSA, via Bonomea 265, 34136 Trieste, Italy
}

(Received 18 March 2019; revised manuscript received 21 February 2020; accepted 24 February 2020; published 20 March 2020)

\begin{abstract}
Much has been learned about universal properties of entanglement entropy (EE) and participation ration (PR) for Anderson localization. We find a new subextensive scaling with system size of the above measures for algebraic localization as noticed in one-dimensional long-range hopping models in the presence of uncorrelated disorder. While the scaling exponent of EE seems to vary universally with the long distance localization exponent of single particle states (SPSs), PR does not show such university as it also depends on the short-range correlations of SPSs. On the other hand, in the presence of correlated disorder, an admixture of two species of SPSs (ergodic delocalized and nonergodic multifractal or localized) are observed, which leads to extensive (subextensive) scaling of EE (PR). Considering typical many-body eigenstates, we obtain above results that are further corroborated with the asymptotic dynamics. Additionally, a finite time secondary slow growth in EE is witnessed only for correlated case while for the uncorrelated case there exists only primary growth followed by the saturation. We believe that our findings from the typical many-body eigenstate would remain unaltered even in the weakly interacting limit.
\end{abstract}

DOI: 10.1103/PhysRevResearch.2.012074

\section{INTRODUCTION}

In one and two dimensions, an arbitrarily weak amount of disorder is sufficient to exponentially localize all eigenstates of a system of noninteracting particles, known as Anderson localization [1-3]. However, correlated disorder in a onedimensional system can lead to the coexistence of exponentially localized and delocalized states, separated by mobility edge (ME) [4-6]. Interestingly, in the presence of interactions a transition between delocalized (ergodic) to many-body localized (MBL) phase can be observed [7,8]. Algebraic localization is another variety of localization that draws significant attention in recent times [9-24]. Entanglement entropy (EE), estimating the bipartite quantum correlations, and participation ratio (PR), quantifying the information about the localization properties of the wave function, happen to be the primary measures of localized and delocalized phases [25-28].

There is an upsurge of studies with disordered models in the presence of long-range hopping that decays with distance $l$ as a power law $1 / l^{a}$ can show algebraic localization [29-32]. Interestingly, Levitov's conjecture [9-11,14] about the absence of localization in a $d$-dimensional model with $a<d$, is violated in one of such a noninteracting long-range model where also single particle states (SPSs) are found to be alge-

Published by the American Physical Society under the terms of the Creative Commons Attribution 4.0 International license. Further distribution of this work must maintain attribution to the author(s) and the published article's title, journal citation, and DOI. braically localized $[9,32,33]$. Recent advancements in experiments with atomic, molecular, and optical systems [34,35], power-law spin interactions with tunable exponent $0<a<3$ can be realized in laser-driven cold atom setup [36,37]. The dipolar $(a=3)$ and van der Waals $(a=6)$ couplings have also been experimentally observed for the ground state of neutral atoms and Rydberg atoms [38-41].

Thanks to the availability of analytical and computational methods, many compelling results have been obtained for EE, e.g., it satisfies the area (volume) law for the exponentially localized (delocalized) phase [25,26,42-47]. Another important diagnostic PR is expected to follow the similar behavior $[27,48]$. While turning into dynamics, EE for clean (disordered) systems show a faster power-law (a slower double log-type) growth with time [28,49,50]. Having known all of these, we here pose the question that what would be the nature of EE and PR in an algebraically localized phase and do they scale identically? Additionally, the big underlying question is to predict the Fock space picture for weakly interacting model by performing a many-body analysis (statics and dynamics) on the noninteracting system.

In particular, we study EE and PR for a noninteracting power-law hopping model in the presence of uncorrelated disorder (referred to as model I), which supports the algebraically localized phase, and correlated disorder (referred to as model II), that contains ME and multifractal phases. We do find that algebraic localization leads to new subextensive scaling of EE and PR with system size for model I, while EE (PR) satisfies extensive (subextensive) scaling for model II. The subextensive nature of PR is possibly a manifestation of multifractality of many-body wave functions in Fock space $[51,52]$. 
Additionally, probing the associated exponents, we can convey that $\mathrm{EE}(\mathrm{PR})$ can capture the long-range (short-range) correlation of SPSs.

\section{MODEL}

We study noninteracting fermions in the one-dimensional (1D) lattice in the presence of a disordered potential. The system is described by the following long-range power-law hopping Hamiltonian:

$$
H=-\sum_{i, j \neq i} \frac{1}{|i-j|^{a}}\left(\hat{c}_{i}^{\dagger} \hat{c}_{j}+\text { H.c. }\right)+\sum_{i} \epsilon_{i} \hat{n}_{i},
$$

where $\hat{c}_{i}^{\dagger}\left(\hat{c}_{i}\right)$ is the fermionic creation (annihilation) operator at site $i, \hat{n}_{i}=\hat{c}_{i}^{\dagger} \hat{c}_{i}$ is the number operator, and $L$ is the size of the system.

We consider two cases here. (1) Model I (with uncorrelated disorder): $\epsilon_{i}$ are chosen randomly from a uniform distribution between $[-W, W]$. In this paper, we choose $W=20$, for which a very tiny fraction of states are delocalized for $a<3 / 2$ and all states are localized for $a>3 / 2$ (see Supplemental Material Ref. [53] for details). It has been shown that the singleparticle wave function $\psi(x)$ of this model displays power-law localization [9] (not exponential) $|\psi(x)|^{2} \sim 1 /\left|x-x_{0}\right|^{\nu}$ in the limit $x \gg x_{0}$, where, $x_{0}$ is the localization center and $v$ is the localization exponent. $v$ shows duality $v(a)=v(2-a)$ around $a=1$ for $0<a<2$ as investigated numerically [33] and analytically [32]. However, we like to point out that near $x_{0}$, in the limit $\left|x-x_{0}\right| \ll L$, the SPSs are completely different in both sides of $a$, while one finds an exponential decay of the wave function for $a>1$, the decay is still algebraic for $a<1$. $a=1$ point has been shown to be critically localized [9]. We note that as $a \rightarrow \infty$, SPS becomes completely exponentially localized with $L \rightarrow \infty$. On the other hand, at $a=0$ the model is exactly solvable and wave functions in the bulk of the spectrum are critically multifractal [54-57]. We note that the algebraic localization is also observed for the Hamiltonian residing in the family of power-law random banded matrix model, e.g., model III [10,11] (see Supplemental Material Ref. [53] for details).

(2) Model II (with correlated disorder): $\epsilon_{i}=h \cos (2 \pi \sigma i+$ $\phi)$, where $\sigma=(\sqrt{5}-1) / 2$ and $\phi$ is an offset chosen from a uniform random distribution [0,1] and closely related to the self-dual quasiperiodic model [19,58]. This seemingly innocent difference has drastic consequences on the physics of this model compared to the previous one. Interestingly, for $a<1$, all SPSs are extended. However, depending on the choice of parameters ( $h$ and $a$ ), there are different phases where ergodic and multifractal (MF) states coexist. We will refer this phase as the MF phase. On the other hand, for $a>1$ there is a coexistence of delocalized and localized SPSs, henc the mobility edge (ME) exists (we will refer to this phase as the ME phase). In either side of $a$, different regimes, denoted by $P_{s}$, are characterized by a fraction $\sigma^{s}<1$ of ergodic SPSs at the bottom of the band and the rest are either localized (for $a>1$ ) or multifractal (for $a \leqslant 1$ ) (see Supplemental Material Ref. [53] for details) [59].

For all calculations in this paper, we restrict ourselves at half-filling. All quantities are obtained after algebraically
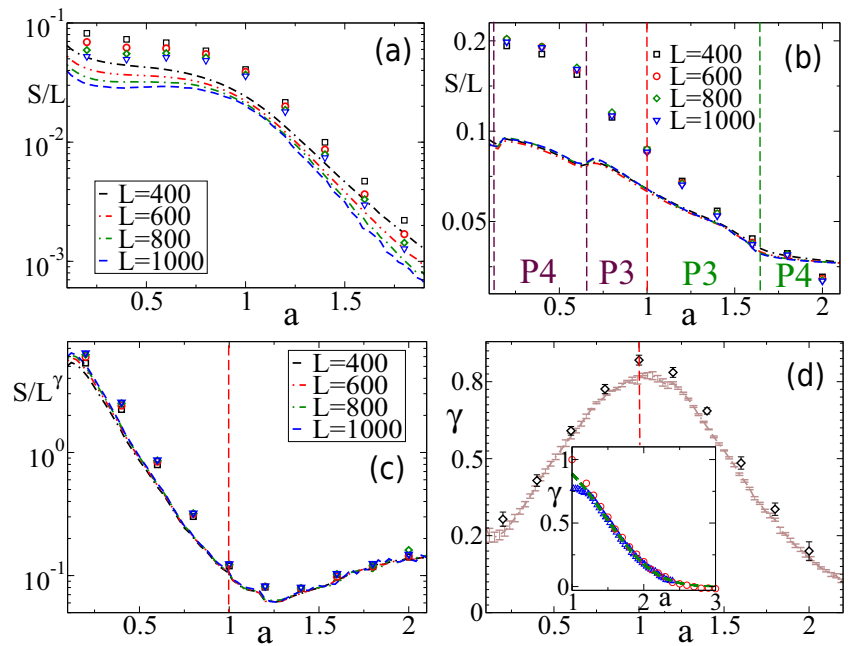

FIG. 1. Entanglement entropy density $S / L$ vs $a$ for model I in (a) and Model II with $h=4.0$ in (b). Here, P3 and P4 represent different multifractal (mobility edge) phases for $a<1(a>1)$. A perfect data collapse is observed in (b) and (c) for different $L$ suggesting volume law of EE in model II and subextensive scaling of $\mathrm{EE}$ in model I, respectively. The variation of subextensive exponent $\gamma(a)$ with $a$ showing duality around $a=1$ is depicted in (d). Lines (symbols) correspond to EE obtained from eigenstates (dynamics). Inset in (d) shows that $\gamma$ for model I (depicted by red circle) and model in Ref. [53] (depicted by blue triangles) behave identically (referenced with stretched exponential, green dashed line) with $a$, which is related to localization exponent $v \simeq 2 a$.

averaging over $10^{3}$ disorder realizations (see Supplemental Material Ref. [53] for details). All time evolution calculations are done starting from an initial product state $\left|\Psi_{0}\right\rangle=$ $\prod_{i=1}^{L / 2} \hat{c}_{2 i}^{\dagger}|0\rangle$.

\section{ENTANGLEMENT ENTROPY}

In this section we will discuss the eigenstate $\mathrm{EE}$ and also the nonequilibrium dynamics of $\mathrm{EE}$ after a global quench starting from the above product state. We note that a typical measure of the entanglement in a quantum system is bipartite von Neumann entanglement entropy $S$ defined as, $S=-\operatorname{Tr}_{A}\left[\rho_{A} \ln \rho_{A}\right]$, where $\rho_{A}=\operatorname{Tr}_{B}|\Psi\rangle\langle\Psi|$ is the reduced density matrix of a subsystem $A$ after dividing the system into two equal adjacent parts $A$ and $B$, both comprised of $L / 2$ sites. $|\Psi\rangle$ is the many-body wave function of the composite system.

\section{A. Eigenstate EE}

For model I, we notice that the typical eigenstate EE [60-63] (for details, see Supplemental Material Ref. [53]) marked by lines in Fig. 1(a) shows the absence of data collapse in $S / L$. However, the data collapse appears when we replace $S / L$ by $S / L^{\gamma(a)}$ with $\gamma(a)<1$ as shown in Fig. 1(c). Interestingly, $\gamma(a)$ exhibits the duality around $a=1: \gamma(a) \simeq$ $\gamma(2-a)$ as shown in Fig. 1(d) (see Supplemental Material Ref. [53] for more details). This duality is the consequence of the duality present in the spatial exponent associated with the algebraically decaying long tail of SPSs for this model in either side of $a<1$ and $a>1$ [64]. Furthermore, the inset 
of Fig. 1(d) suggests that the exponent $\gamma$ follows a universal behavior with spatial exponent $a$ as long as SPSs are algebraically localized. The subextensive $L^{\gamma}$ law can be naively understood from the spatial algebraic structure of SPSs. The total probability of finding a particle at any site $\in B$ while its localization center is at site $x_{0} \in A$ becomes $p \sim \sum_{x \in B} \mid x-$ $\left.x_{0}\right|^{-2 a} \sim L^{1-2 a} f\left(x_{0}, a, L\right)$ where $f$ is a nonlinear function of $x_{0}, a, L$. This type of fractional scaling with $L$ is absent for exponentially localized SPSs where $p \sim \xi f\left(x_{0}, \xi, L\right)$ and $\xi$ being the localization length. Moreover, in the many-body case, EE becomes a complex function of $x_{0}$ as the different SPSs have different localization centers. The absence of the coherent length scale $\xi$ in algebraic localization can lead to nontrivial subextensive behavior in many-body EE.

On the other hand, Fig. 1(b) shows the presence of data collapse in $S / L$ for model II. There exists a fraction of delocalized ergodic phase yielding $S$ extensively. Even though, in both the MF $(a<1)$ and ME phase $(a>1), S \sim L$ [as shown in Fig. 1(b)], one can distinguish them by their corresponding numerical values of $S / L$. This is higher in the MF phase compared to the ME phase because all SPSs are essentially delocalized in the MF phase. Moreover, in both sides of $a=1$, the transition between different $P_{s}$ phases are clearly visible. The volume law of eigenstate EE has also been recently observed for the 1D short-range noninteracting model in the presence of correlated disorder, where there exists a mobility edge in the single particle spectrum $[64,65]$.

\section{B. Asymptopic EE}

We shall now extensively investigate the scaling of asymptotic saturation value $S(L, t \rightarrow \infty) \equiv S_{\infty}$ with $a$ as marked by symbols, starting from an initial product state $\left|\Psi_{0}\right\rangle$. Figures 1(b) and 1(a) show the presence of data collapse in $S_{\infty} / L$ for model II and the absence of it in model I, respectively, for different values of $L$. Similar to the typical eigenstates, we here in the dynamics find the subextensive (extensive) nature of EE and duality in $\gamma$ for model I (model II) as shown in Figs. 1(c) and 1(d) [Fig. 1(b)]. However, the proportionality factor associated with $S / L$ changes from its eigenstate value. Since these are noninteracting systems, we expect that $S_{\infty}$ obtained from dynamics should show similar behavior as typical eigenstates. Note that interaction leads to the dephasing mechanism via scattering in the system and hence, for the interacting system the above expectation may not hold true. MBL systems are one such example, where we see that the EE of many-body eigenstates obey area law, however, $S_{\infty} \sim L[26,44]$. Since, for both models, we do not have a parameter regime where all states are ergodic (delocalized), hence, $S_{\infty} / L$ and also the eigenstates EE density always become less than the Page value [66]. The EE associated with the midspectrum eigenstates of a generic interacting nonintegrable systems obeying ETH $[46,67,68]$, satisfy this bound.

\section{Finite time rise}

Having studied finite-size scaling of aymptotic EE $S_{\infty}$, now we analyze the finite time growth of EE. The results are shown for model I in Figs. 2(a) and 2(b). We observe for $a \leqslant 1$, a power-law rise occurs, $S \sim t^{\alpha}$. This growth exponent
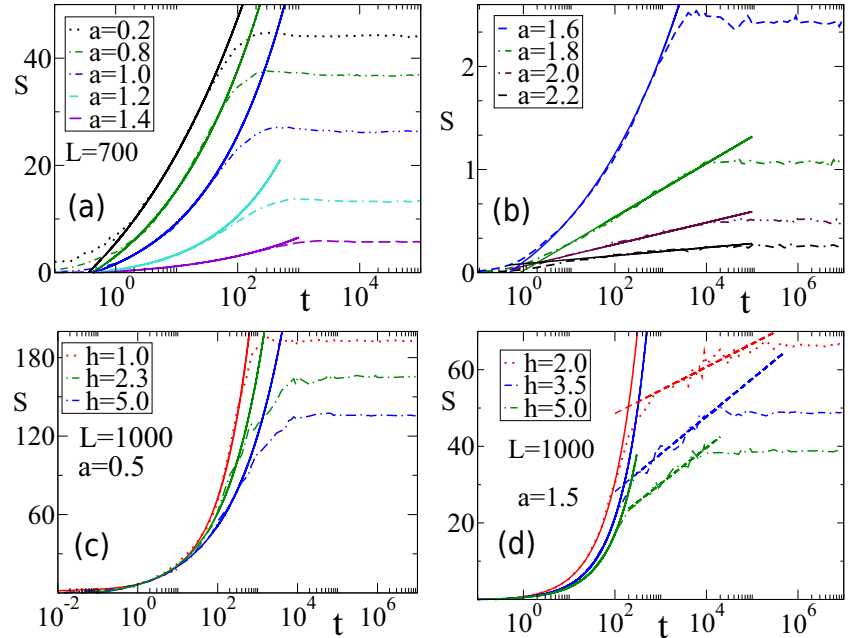

FIG. 2. Time evolution of entanglement entropy $S(t)$ for model I is shown for $0.2 \leqslant a \leqslant 1.4$ in (a) and for $1.6 \leqslant a \leqslant 2.2$ in (b). Solid (dashed) lines correspond to power-law $t^{\alpha}$-fit $[\log (t)$-fit $]$. Same results are shown for model II in (c) with $a=0.5$ and in (d) with $a=1.5$.

$\alpha$ becomes larger near the point $a=1$ (see Supplemental Material Ref. [53] for details). For $a>1$, the growth exponent $\alpha$ decreases and for $a \sim 2$, EE shows a logarithmic rise. Note that in the case of Hamiltonian (1) even without disorder, the initial growth of $S(t)$ is sublinear in $t$ [53,69]. Since, SPSs behave differently in either side of $a$; the presence of the rapid fall of the single particle wave function near the localization center causes the relatively slow rise of EE for $1<a<2$ compared to the $a<1$ regime [29]. We note that our power-law growth of EE resembles with the out of time ordered correlator showing a deviation from light-cone-like behavior in the context of long-range models [22].

Turning into model II as shown in Figs. 2(c) and 2(d), one can see a fast power-law rise $\left(S \sim t^{\alpha_{1}}\right)$ with $\alpha_{1}<1$ followed by a much slower rise in EE. We observe the value of $\alpha_{1}$ is larger for $a>1$ in comparison to the $a<1$ case. We also note that in the MF phase, the growth exponent depends on $P_{s}$ phases, whereas, in the ME phase, $\alpha$ remains almost same in different $P_{s}$ phases (see Supplemental Material Ref. [53] for details). This is presumably the consequence of the fact that the spatial structure of the mutifractal SPS are different in different $P_{s}$ phases. Contrastingly, for $a>1$ even in different $P_{s}$ ME phases, the spatial structure of localized states wave function are different from $a<1$. For $a>1$, the long time slow growth, visible in a reasonably large time window, is found to be logarithmic.

On the other hand, for $a<1$, we see a similar secondary slow rise of $S$ since the time window is much smaller we cannot comment on it whether this is a power law with exponent $\alpha_{2}<\alpha_{1}$ or logarithmic.

One can naively connect our results with the noninteracting central site model [70], where multifractality, appearing due to the coupling of a single central bound state with all Anderson localized states, can give rise to a slow logarithmic growth in entanglement dynamics. Previously, the logarithmic growth of $S$ was thought to be a unique feature of MBL systems [44,71]. 

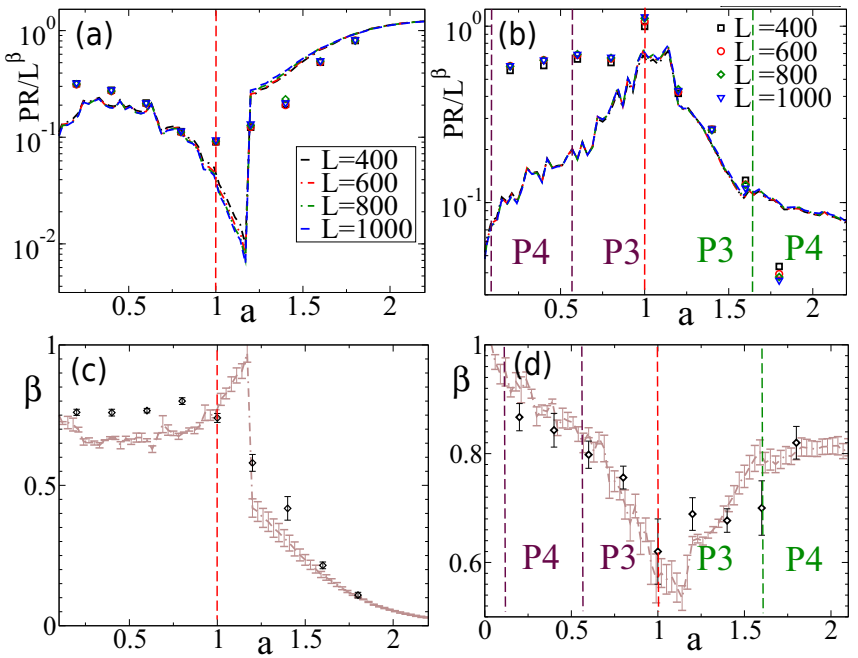

FIG. 3. The subextensive scaling of PR is shown by the data collapse of PR/L $L^{\beta}$ vs $a$ for model I in (a) and model II in (b). The variation of $\beta(a)$ with $a$ for model I and II are depicted in (c) and (d), respectively. Lines (symbols) correspond to PR obtained from eigenstates (dynamics). $h=4.0$ is considered for model II.

Interestingly, our results indicate that the presence of two different types of SPS, there exists a secondary slow rise in the finite time evolution of $S$ for model II; this growth is completely missing for the uncorrelated disordered model I.

Based on our analysis of the finite time EE in models I and II, we can convey that the sublinear temporal growth $S \sim t^{\alpha\left(\alpha_{1}\right)}$ is related to the detail (structure and fraction of delocalized states) of SPSs in these systems: (1) This is apparently evident form the behavior of $S$ for $a<3 / 2$ in model I, and (2) for model II, $\alpha_{1}$ remains unaltered with $a$ as long as one stays inside a fixed $P_{s}$ phase. However, the value of $\alpha$ interestingly changes as one varies $a$; similarly, $\alpha_{1}$ changes as one goes from MF side to ME side even within the same $P_{s}$ phase. A finite fraction of delocalized SPSs can also cause the two-stage growth of EE in model II. On the other hand, this fraction becomes vanishingly small for model I originating from the single stage growth (see Supplemental Material Ref. [53] for a detailed analysis).

\section{PARTICIPATION RATIO}

Having examined the scaling of eigenstate EE and $S_{\infty}$ with $L$, in the similar spirit, we now look for the scaling of eigenstate PR and the saturation values of PR (designated by $\mathrm{PR}_{\infty}$ ). We shall use the definition of many-body $\mathrm{PR}$ as introduced in Ref. [46]. It is defined for the half-filling case as, $\mathrm{PR}=L\left[2 \sum_{\alpha=1}^{L} n_{\alpha} \sum_{j=1}^{L}\left|\phi_{\alpha}(j)\right|^{4}\right]^{-1}$, where, $\left|\phi_{\alpha}\right\rangle$ and $n_{\alpha}$ are eigenvectors and eigenvalues of the one-body density matrix $\rho_{i j}=\left\langle\hat{c}_{i}^{\dagger} \hat{c}_{j}\right\rangle$, respectively. PR $\sim L$ for delocalized ergodic systems and $\mathrm{PR} \sim \xi$ for exponentially localized many-body states $[27,48]$.

We study the characteristics of $\mathrm{PR}_{\infty}$ and eigenstate $\mathrm{PR}$ in Fig. 3. From the data collapse of $\mathrm{PR} / L^{\beta}$ as a function of $a$ with different system sizes [see Figs. 3(a) and 3(b)], we show for both the models that PR exhibits a subextensive scaling with $L$ (see Supplemental Material Ref. [53] for detail). To analyze the exponent $\beta$ more concretely, we show the variation of $\beta$ with $a$ for models I and II in Figs. 3(c) and 3(d), respectively. $\beta$ remains fixed at a higher value for $a<1$ while it decreases monotonically for $a>1$ for model I. Very surprisingly, unlike the EE, PR does not exhibit any duality with $a$ around $a=$ 1. The reason being PR is a local quantity, it is not able to capture the duality of SPSs in the long-distance scale where the power-law tail is observed in either side of $a=1$. Precisely, PR accounts for the short-distance behavior of SPSs where exponential and algebraic decay are present for $a>1$ and $a<1$, respectively, hence, $\beta$ is completely asymmetric around $a=1$.

On the other hand, for model II, $\beta$ shows a kind of symmetric behavior around $a=1$ (see Fig. 3). This result may be counterintuitive in the sense that phases in both sides of $a=1$ are completely different, i.e., the MF phase for $a<1$ and ME phase for $a>1$. Multifractal SPSs in this model have a form of a multiple sharp peak on the top of the almost flat background in contrast to the ergodic delocalized SPSs that are extended all over the lattice [53]. The structure of SPSs for the MF phase is kind of similar to the exponentially localized SPSs having only one peak and the background is suppressed exponentially with distance from that peak. Since $\mathrm{PR}$ is an inappropriate measure to identify the fine-tuned long distance structure of SPSs for ME and MF phases, we find similar behavior of $\beta$ in either side of $a$. However, we note that $\beta$ is much closer to 1 in the MF phase compared to the ME phase. Moreover, from the variation of $\beta$ with $a$, we can roughly identify different $P_{s}$ phases in either side of $a=1$ [see Fig. 3(d)]. Moreover, we note that in the calculation with typical eigenstates, we discard a few bottom spectrum delocalized states to minimize their effect (see Supplemental Material Ref. [53] for details). On the other hand, all the energy states come automatically into the noneignestate dynamics. We believe that this is the origin of the apparent dissimilarities between the predictions from the typical eigenstate and long time dynamics as observed in Figs. 1 and 3.

\section{CONCLUSION}

We summarize our main results in Table I. One of the most intriguing findings is to show the subextensive scaling in EE and PR, when SPSs are algebraically localized as observed in model I. This is firmly evident from both the eigenstate and long time dynamics. The absence of length scale thus imprints its signature unlike the exponentially localized phase. Moreover, these behaviors are not the artifact of the delocalized states present in model I (at least for $a<3 / 2$ ) as the number

TABLE I. Summary of the main differences between different phases in the noninteracting long-range systems. We note that $\beta$ and $\gamma$ are model-dependent exponents.

\begin{tabular}{lll}
\hline \hline SPS & \multicolumn{1}{c}{$S_{\infty}$} & \multicolumn{1}{c}{$P R_{\infty}$} \\
\hline Exponential localization & $L^{0}$ & $L^{0}$ \\
Algebraic localizaion & $L^{\gamma}, \gamma<1$ & $L^{\beta}, \beta<1$ \\
Ergodic (delocalized) & $L$ & $L$ \\
Multifractal (nonergodic) & $L$ & $L^{\beta}, 0<\beta \leqslant 1$ \\
Mobility-edge & $L$ & $L^{\beta}, 0<\beta \leqslant 1$ \\
\hline \hline
\end{tabular}


of such states has measure zero for $L \rightarrow \infty[33,55,72]$. Turning to model II, asymptotic and eigenstate EE both obey volume law due to the presence of ergodic SPSs; however, interestingly, the proportionality factors change in different $P_{s}$ phases. The adiabatic connectivity allows us to conjecture that algebraically localized quasilocal integrals of motion would survive even in the weakly interacting limit $[23,26,56,73]$ and hence, the eigenstate EE scaling should remain unaltered even in the above limit. One might not expect the similar scaling of asymptotic EE, obtained from the long time dynamics, due to the dephasing mechanism caused by the interaction.

Our study further reveals the connection between the exponents ( $\gamma$ for EE and $\beta$ for PR), and the spatial structure of the SPSs. The EE is maximally governed by the long-distance nature of the SPSs and thus the duality in $\gamma$ is closely connected to the duality of the localization exponent $v$ as noticed for model I [33]. Moreover, $\gamma$ follows a universal behavior as far as the algebraically localized SPSs are concerned. In contrary, PR captures the short-distance nature of correlation leading to the fact that exponent $\beta$ does not show duality around $a=1$. The short-distance behavior of SPSs are very different for $a<1$ and $a>1$ for model I. Surprisingly, model II shows duality-like behavior within a small window around $a=1$. This can be related to the peculiar spatial distribution of multifractal SPSs at short distance (see Supplemental Material Ref. [53] for details).

Another important contribution of our work is to show how the structure of SPSs can influence the finite time rise of EE. An unprecedented two-stage growth of EE for model II is exclusively observed while model I exhibits single-stage growth. The secondary rise in EE for model II might be related to the fact that there exists a finite fraction of two types of SPSs, i.e., multifractal and delocalized or localized and delocalized. The initial algebraic temporal growth is common in both the models. Recent studies also find signatures of temporal power-law growth of EE in long range interacting models $[23,74]$. The connection between the temporal powerlaw growth of EE and algebraical SPSs (LIOM) for the noninteracting (interacting) model is still an open field of research. Given the experimental realizability of spin models [38-41], we believe that our study would initiate a plethora of work in this direction.

\section{ACKNOWLEDGMENTS}

The authors thank P. Calabrese and L. Vidmar for reading the manuscript and for several comments. The authors also thank the anonymous referee for useful comments.
[1] P. W. Anderson, Phys. Rev. 109, 1492 (1958).

[2] E. Abrahams, P. W. Anderson, D. C. Licciardello, and T. V. Ramakrishnan, Phys. Rev. Lett. 42, 673 (1979).

[3] P. A. Lee and T. Ramakrishnan, Rev. Mod. Phys. 57, 287 (1985).

[4] S. Das Sarma, S. He, and X. C. Xie, Phys. Rev. B 41, 5544 (1990).

[5] S. Ganeshan, J. H. Pixley, and S. Das Sarma, Phys. Rev. Lett. 114, 146601 (2015).

[6] R. Modak and S. Mukerjee, Phys. Rev. Lett. 115, 230401 (2015).

[7] D. Basko, I. Aleiner, and B. Altshuler, Ann. Phys. (NY) 321, 1126 (2006).

[8] I. Gornyi, A. Mirlin, D. Polyakov, and A. Burin, Ann. Phys. 529, 1600360 (2017).

[9] A. L. Burin and L. A. Maksimov, JETP Lett. 50, 338 (1989).

[10] L. S. Levitov, Europhys. Lett. 9, 83 (1989).

[11] L. S. Levitov, Phys. Rev. Lett. 64, 547 (1990).

[12] A. D. Mirlin, Y. V. Fyodorov, F.-M. Dittes, J. Quezada, and T. H. Seligman, Phys. Rev. E 54, 3221 (1996).

[13] A. Rodríguez, V. A. Malyshev, and F. Domínguez-Adame, J. Phys. A: Math. Gen. 33, L161 (2000).

[14] A. V. Malyshev, V. A. Malyshev, and F. Domínguez-Adame, Phys. Rev. B 70, 172202 (2004).

[15] A. D. Mirlin and F. Evers, Phys. Rev. B 62, 7920 (2000).

[16] N. Y. Yao, C. R. Laumann, S. Gopalakrishnan, M. Knap, M. Müller, E. A. Demler, and M. D. Lukin, Phys. Rev. Lett. 113, 243002 (2014).

[17] A. L. Burin, Phys. Rev. B 91, 094202 (2015).

[18] A. L. Burin, Phys. Rev. B 92, 104428 (2015).

[19] S. Gopalakrishnan, Phys. Rev. B 96, 054202 (2017).
[20] R. M. Nandkishore and S. L. Sondhi, Phys. Rev. X 7, 041021 (2017).

[21] K. S. Tikhonov and A. D. Mirlin, Phys. Rev. B 97, 214205 (2018).

[22] D. J. Luitz and Y. Bar Lev, Phys. Rev. A 99, 010105(R) (2019).

[23] G. De Tomasi, Phys. Rev. B 99, 054204 (2019).

[24] S. Roy and D. E. Logan, SciPost Phys. 7, 042 (2019).

[25] S. Iyer, V. Oganesyan, G. Refael, and D. A. Huse, Phys. Rev. B 87, 134202 (2013).

[26] M. Serbyn, Z. Papić, and D. A. Abanin, Phys. Rev. Lett. 110, 260601 (2013).

[27] G. L. Celardo, R. Kaiser, and F. Borgonovi, Phys. Rev. B 94, 144206 (2016).

[28] R. Vosk and E. Altman, Phys. Rev. Lett. 110, 067204 (2013).

[29] R. Singh, R. Moessner, and D. Roy, Phys. Rev. B 95, 094205 (2017).

[30] J. T. Cantin, T. Xu, and R. V. Krems, Phys. Rev. B 98, 014204 (2018).

[31] T. Botzung, D. Vodola, P. Naldesi, M. Müller, E. Ercolessi, and G. Pupillo, Phys. Rev. B 100, 155136 (2019).

[32] P. Nosov, I. Khaymovich, and V. Kravtsov, Phys. Rev. B 99, 104203 (2019).

[33] X. Deng, V. E. Kravtsov, G. V. Shlyapnikov, and L. Santos, Phys. Rev. Lett. 120, 110602 (2018).

[34] M. Kastner, Phys. Rev. Lett. 104, 240403 (2010).

[35] Z.-X. Gong, M. Foss-Feig, S. Michalakis, and A. V. Gorshkov, Phys. Rev. Lett. 113, 030602 (2014).

[36] P. Richerme, Z.-X. Gong, A. Lee, C. Senko, J. Smith, M. FossFeig, S. Michalakis, A. V. Gorshkov, and C. Monroe, Nature 511, 198 (2014).

[37] J. W. Britton, B. C. Sawyer, A. C. Keith, C.-C. J. Wang, J. K. Freericks, H. Uys, M. J. Biercuk, and J. J. Bollinger, Nature 484, 489 (2012). 
[38] S. Baier, D. Petter, J. H. Becher, A. Patscheider, G. Natale, L. Chomaz, M. J. Mark, and F. Ferlaino, Phys. Rev. Lett. 121, 093602 (2018).

[39] Y. Tang, W. Kao, K.-Y. Li, and B. L. Lev, Phys. Rev. Lett. 120, 230401 (2018).

[40] H. Weimer, R. Löw, T. Pfau, and H. P. Büchler, Phys. Rev. Lett. 101, 250601 (2008)

[41] M. Viteau, P. Huillery, M. G. Bason, N. Malossi, D. Ciampini, O. Morsch, E. Arimondo, D. Comparat, and P. Pillet, Phys. Rev. Lett. 109, 053002 (2012).

[42] F. Iglói, Z. Szatmári, and Y.-C. Lin, Phys. Rev. B 85, 094417 (2012).

[43] K. Agarwal, S. Gopalakrishnan, M. Knap, M. Müller, and E. Demler, Phys. Rev. Lett. 114, 160401 (2015).

[44] J. H. Bardarson, F. Pollmann, and J. E. Moore, Phys. Rev. Lett. 109, 017202 (2012)

[45] S. Ghosh, A. Acharya, S. Sahu, and S. Mukerjee, Phys. Rev. B 99, 165131 (2019).

[46] S. Bera, H. Schomerus, F. Heidrich-Meisner, and J. H. Bardarson, Phys. Rev. Lett. 115, 046603 (2015).

[47] Y. Zhao, F. Andraschko, and J. Sirker, Phys. Rev. B 93, 205146 (2016).

[48] A. Rodríguez, V. A. Malyshev, G. Sierra, M. A. MartínDelgado, J. Rodríguez-Laguna, and F. Domínguez-Adame, Phys. Rev. Lett. 90, 027404 (2003).

[49] P. Calabrese and J. Cardy, J. Stat. Mech. (2016) 064003.

[50] P. Calabrese and J. Cardy, J. Stat. Mech. (2005) P04010.

[51] A. D. Mirlin, Y. V. Fyodorov, A. Mildenberger, and F. Evers, Phys. Rev. Lett. 97, 046803 (2006).

[52] F. Evers and A. D. Mirlin, Rev. Mod. Phys. 80, 1355 (2008).

[53] Please see Supplemental Material at http://link.aps.org/ supplemental/10.1103/PhysRevResearch.2.012074 for more information.

[54] H. K. Owusu, K. Wagh, and E. A. Yuzbashyan, J. Phys. A: Math. Theor. 42, 035206 (2008).

[55] A. Ossipov, J. Phys. A: Math. Theor. 46, 105001 (2013).
[56] R. Modak, S. Mukerjee, E. A. Yuzbashyan, and B. S. Shastry, New J. Phys. 18, 033010 (2016).

[57] J. A. Scaramazza, B. S. Shastry, and E. A. Yuzbashyan, Phys. Rev. E 94, 032106 (2016).

[58] J. Biddle, D. J. Priour, B. Wang, and S. Das Sarma, Phys. Rev. B 83, 075105 (2011).

[59] X. Deng, S. Ray, S. Sinha, G. V. Shlyapnikov, and L. Santos, Phys. Rev. Lett. 123, 025301 (2019).

[60] L. Vidmar, L. Hackl, E. Bianchi, and M. Rigol, Phys. Rev. Lett. 121, 220602 (2018).

[61] L. Vidmar and M. Rigol, Phys. Rev. Lett. 119, 220603 (2017).

[62] L. Vidmar, L. Hackl, E. Bianchi, and M. Rigol, Phys. Rev. Lett. 119, 020601 (2017).

[63] L. Hackl, L. Vidmar, M. Rigol, and E. Bianchi, Phys. Rev. B 99, 075123 (2019).

[64] D.-L. Deng, S. Ganeshan, X. Li, R. Modak, S. Mukerjee, and J. Pixley, Ann. Phys. (NY) 529, 1600399 (2017).

[65] R. Modak, S. Ghosh, and S. Mukerjee, Phys. Rev. B 97, 104204 (2018).

[66] D. N. Page, Phys. Rev. Lett. 71, 1291 (1993).

[67] L. D'Alessio, Y. Kafri, A. Polkovnikov, and M. Rigol, Adv. Phys. 65, 239 (2016).

[68] M. Rigol and M. Srednicki, Phys. Rev. Lett. 108, 110601 (2012).

[69] A. S. Buyskikh, M. Fagotti, J. Schachenmayer, F. Essler, and A. J. Daley, Phys. Rev. A 93, 053620 (2016).

[70] D. Hetterich, M. Serbyn, F. Domínguez, F. Pollmann, and B. Trauzettel, Phys. Rev. B 96, 104203 (2017).

[71] M. Žnidarič, T. Prosen, and P. Prelovšek, Phys. Rev. B 77, 064426 (2008).

[72] F. A. B. F. de Moura, A. V. Malyshev, M. L. Lyra, V. A. Malyshev, and F. Domínguez-Adame, Phys. Rev. B 71, 174203 (2005).

[73] J. Z. Imbrie, J. Stat. Phys. 163, 998 (2016).

[74] A. Safavi-Naini, M. L. Wall, O. L. Acevedo, A. M. Rey, and R. M. Nandkishore, Phys. Rev. A 99, 033610 (2019). 